\title{
Invariants in Optimal Control: An Exact Solution of the Optimal Stabilization Problem
}

\author{
Gennadii V. Kondratiev ${ }^{1, \mathrm{a} *}$ \\ ${ }^{1}$ Department of Mathematics and Mathematical Education, K. Minin State Pedagogical University, \\ Nizhniy Novgorod, Russia
}

agennadii.kondratiev@gmail.com

Keywords: control system, integral functional, phase space, optimal feedback, canonical transformations, geometric invariants

\begin{abstract}
The stabilizing optimal feedback is a function on the separatrix of stable points of the associated Hamiltonian system. Three geometric objects - the symplectic form, Hamiltonian vector field, and Lyapunov function, generating the separatrix — are invariantly attached to the optimal control system under canonical transformations of the phase space. The separatrix equations can be written in terms of these invariants through invariant operations. There is a computable representative of the equivalence class, containing the original system. It is its linear approximation system at the stable point.
\end{abstract}

Subject Classification Numbers: 49J15, 49J21, 37J05, 37J15.

\section{Introduction}

The problem of optimal stabilization is normally reformulated in terms of the associated Hamiltonian system.

The separatrix of stable points with respect to the origin of the Hamiltonian system is a Lagrangian manifold of the maximal dimension, which consists of all integral curves going to the origin from a maximal neighbourhood. The standard procedure to compute a Lagrangian manifold is the Cauchy characteristics method, extending an initial Lagrangian surface transversal to the integral curves of the Hamiltonian vector field along them to a Lagrangian surface of the dimension larger by one. In the optimal stabilization problem there is no known tranversal surface of this type. The Cauchy characteristics method fails due to the incorrect initial conditions - only one point, the origin of the phase space, is known.

In such cases, a powerful method of invariants associated to the object can be successfully used.

Invariants are just functions constructed from the base intrinsic objects using operations, invariant under admissible maps, such as the canonical transformations in Hamiltonian Mechanics.

Having invariants on the orbit of the transformation group and the values of them for a canonical representative of the orbit, one automatically has these invariants and their values, distributed over all the orbit points.

The paper proposes to use the following invariants - the symplectic form, Hamiltonian vector field, separatrix of stable points, generating Lyapunov function of the separatrix - to obtain exact formulas of the separatrix and optimal feedback. A canonical representative whose invariants are computable is the linear approximation of the system at the stable point.

The statement and analysis of the optimal stabilization problem from the point of Hamiltonian Mechanics can be found in [1], the notions of Hamiltonian Mechanics in [2], vector fields and differential forms in [3], general ideas on local invariants of differential-geometric objects in [4, 5, 6, 7].

It should be noticed that the method of invariants is a powerful tool and undeservedly forgotten. The author used it in absolutely different areas such as Statistics and Data Analysis with the same ideology although with a different category-theoretic approach $[8,9]$ to get a high precision prediction tool and invariants of data. 


\section{The Problem Formulation}

A smooth optimal stabilization problem is formulated as the following. For a control system

$$
\dot{x}=f(x, u), \quad x \in \mathbb{R}^{n}, u \in \mathbb{R}^{m}, f(0,0)=0,
$$

and a functional

$$
J=\int_{0}^{\infty} \omega(x, u) d t, \omega(x, u) \geq 0, \omega(0,0)=0,
$$

where: $f$ and $\omega$ are smooth functions, $\omega$ is nondegenerated at 0 , that is its quadratic approximation at $x=0, u=0$ is represented by a matrix with a nonzero determinant,

find a smooth stabilizing optimal feedback $u=u_{\text {opt }}(x)$, that is, a smooth function, providing stability of the origin $x=0$ of the control system 1 with $u=u_{o p t}(x)$ and minimum of the functional 2 in the sense, that

$$
V\left(x_{0}\right)=\int_{0}^{\infty} \omega\left(x_{\text {opt }}\left(t, x_{0}\right), u_{\text {opt }}\left(x_{\text {opt }}\left(t, x_{0}\right)\right)\right) d t \leq \int_{0}^{\infty} \omega\left(x\left(t, x_{0}\right), u\left(x\left(t, x_{0}\right)\right)\right) d t
$$

for each point $x_{0} \in U, U \subset \mathbb{R}^{n}$ is a neighbourhood of $x=0$,

where $x\left(t, x_{0}\right)$ is the trajectory of system 1 with $u=u(x)$, starting at point $x(0)=x_{0}$. Function $u(x)$ is required to stabilize the system 1 at the origin.

Both sides of inequality 3 are Lyapunov functions of the corresponding systems of ODEs.

It is well-known, that when the linear approximation system of the system 1 at $x=0, u=0$ is controllable, the problem $1,2,3$ has a unique solution $u_{\text {opt }}: W \rightarrow \mathbb{R}^{m}$ on a maximal neighbourhood $W$ of the origin $x=0$.

The function $u_{\text {opt }}(x), x \in W$, is a fucntion obtained as

$$
u_{\text {opt }}(x)=\left.u_{\text {opt }}\left(x^{i}, V_{j}\right)\right|_{V_{j}=V_{x}(x)}=\left.\operatorname{Arg}\left(\min _{u \in \mathbb{R}^{m}}\left(\sum_{i=1}^{n} f^{i}(x, u) \cdot V_{i}+\omega(x, u)\right)\right)\right|_{V_{j}=V_{x j}(x)},
$$

where $V_{x^{j}}=\frac{\partial V}{\partial x^{j}}, j=1, \ldots, n$, are the first derivatives of the Lyapunov function $V(x)$ as in 3 which satisties the Hamilton-Jacobi equation

$$
\phi\left(x^{i}, V_{x^{j}}\right)=\sum_{i=1}^{n} f^{i}(x, u) \cdot V_{x^{i}}+\left.\omega(x, u)\right|_{u=u_{o p t}\left(x^{i}, V_{x^{j}}\right)}=0 .
$$

The function $\phi\left(x^{i}, V_{j}\right)$ is the Hamiltonian of the problem. Coordinate functions $x^{i}, i=1, \ldots, n$, and $V_{j}, j=1, \ldots, n$, are respectively coordinates and impulses in the phase space $\mathbb{R}^{2 n}$.

\section{Reformulation of the Problem in the Phase Space}

There are three main geometric objects in the phase space associated to the problem $1,2,3$. They are

the symplectic 2 -form

$$
\Omega=d V_{1} \wedge d x^{1}+\cdots+d V_{n} \wedge d x^{n}
$$

the Hamiltonian vector field 


$$
I d \phi=\frac{\partial \phi}{\partial V_{1}} \frac{\partial}{\partial x^{1}}+\cdots+\frac{\partial \phi}{\partial V_{n}} \frac{\partial}{\partial x^{n}}-\frac{\partial \phi}{\partial x_{1}} \frac{\partial}{\partial V^{1}}-\cdots-\frac{\partial \phi}{\partial x_{n}} \frac{\partial}{\partial V^{n}},
$$

and the separatrix $S^{+}$of stable points with respect to the origin $x^{i}=0, V_{j}=0, i, j=1, \ldots, n$, of the Hamiltonian vector field 7.

$$
\begin{aligned}
S^{+}= & \left\{\left(x^{1}, \ldots, x^{n}, V_{1}, \ldots, V_{n}\right) \in \mathbb{R}^{2 n} \mid 0 \in \mathbb{R}^{2 n}\right. \text { is the limit point } \\
& \text { of the integral curve of } \left.I d \phi \text { with the initial point }\left(x^{i}, V_{j}\right)\right\} .
\end{aligned}
$$

The field 7 is obtained from $d \phi$ as a unique vector field such that the substitution brings $i_{I d \phi} \Omega=d \phi$. The optimal stabilizing feedback $u_{\text {opt }}\left(x^{i}, V_{j}\right)$ as in 4 is a vector-valued function on $S^{+}$:

$$
u_{\text {opt }}(x)=\left.u_{\text {opt }}\left(x^{1}, \ldots, x^{n}, V_{1}, \ldots, V_{n}\right)\right|_{S^{+}} .
$$

So, the problem is to calculate the separatrix $S^{+}$.

The object $S^{+}$is a Lagrangian manifold, that is, one nullifying the symplectic form $\left.\Omega\right|_{S^{+}}=0$, with the generating Lyapunov function $V(x)$. The manifold $S^{+}$is given as $V_{j}=V_{x^{j}}(x), j=1, \ldots, n$.

Since, there is only one known point of the separatrix - the origin of the phase space $\mathbb{R}^{2 n}$ - the method of Cauchy characteristics does not work.

The generating function of a Lagrangian manifold is determined uniquely up to a constant modulo the equations of the Lagrangian manifold, as in the following exact sequence

$$
V(x) \bmod S^{+} \stackrel{d}{\longmapsto} \sum_{i=1}^{n} V_{i} d x^{i} \bmod S^{+} \stackrel{d}{\longmapsto} \Omega \bmod S^{+}=0
$$

\section{The Method of Invariants}

The method is based on two assumptions. The first one is specific, that for any small $\varepsilon$ - neighbourhood $U_{\varepsilon}$ of the orgin $0 \in \mathbb{R}^{2 n}$ there exists $t>0$ such that the preimage $e^{-t \cdot I d \phi}\left(U_{\varepsilon}\right)$ of the flow, generated by the field $I d \phi$, contains the intersection of the separatrix $S^{+}$of stable points of the vector field $I d \phi$ and an arbitrarily big chosen neighbourhood of the origin $0 \in \mathbb{R}^{2 n}$. For any $t \in \mathbb{R}$, this flow is a canonical transformation, preserving the symplectic form 6 , vector field 7 , and mapping the separatrix $S^{+}$to its small part around $0 \in \mathbb{R}^{2 n}$. It follows, that the triple $\left(\Omega, I d \phi, S^{+}\right)$is isomorphic to the similar one $\left(\Omega, I d \phi_{l}, S_{l}^{+}\right)$, where $I d \phi_{l}$ is a linearization of the field $I d \phi$ at point $0 \in \mathbb{R}^{2 n}$, and $S_{l}^{+}$is a separatrix of stable points of the field $I d \phi_{l}$.

The second assumption is general. Any consequences of the triple, obtained by operations not alternating under admissible transformations, make intrinsic sense of the triple.

The vector field $I d \phi$ has a stable point at the origin $0 \in \mathbb{R}^{2 n}$. When this point is nondegenerated, it is hyperbolic one and two separatrices $S^{+}$and $S^{-}$of dimension $n$ go through it, consisting respectively of the points approaching to the origin and going away of it. The phase portrait of the vector field $I d \phi$ is equivalent to one of the linearization $I d \phi_{l}$ in a maximal neighbourhood of the origin $0 \in \mathbb{R}^{2 n}$.

The field $I d \phi_{l}$ has two corresponding linear separatrices $S_{l}^{+}$and $S_{l}^{-}$of the form $V_{i}=\sum_{j=1}^{n} A_{i j} x^{j}$, $i=1, \ldots, n$, where the symmetric matrix $A=A^{T}$ satisfies to the Riccati equation, obtained in the following way.

The quadratic form

$$
\phi_{l}(\bar{V}, \bar{x})=[\bar{V}, \bar{x}]\left(\begin{array}{ll}
H_{1} & H_{2} \\
H_{3} & H_{4}
\end{array}\right)[\bar{V}, \bar{x}]^{T}=\bar{V} H_{1} \bar{V}^{T}+\bar{V} H_{2} \bar{x}^{T}+\bar{x} H_{3} \bar{V}^{T}+\bar{x} H_{4} \bar{x}^{T},
$$


where $H_{1}=H_{1}^{T}, H_{3}=H_{2}^{T}, H_{4}=H_{4}^{T}$ are $n \times n$-matrices, $\bar{V}=\left(V_{1}, \ldots, V_{n}\right), \bar{x}=\left(x^{1}, \ldots, x^{n}\right)$ are $n$-vectors, vanishes on the separatrices $S^{+}, S^{-}$, that is,

$$
\bar{x} A^{T} H_{1} A \bar{x}^{T}+\bar{x} A^{T} H_{2} \bar{x}^{T}+\bar{x} H_{3} A \bar{x}^{T}+\bar{x} H_{4} \bar{x}^{T}=\bar{x}\left(A^{T} H_{1} A+A^{T} H_{2}+H_{2}^{T} A+H_{4}\right) \bar{x}^{T}=0 .
$$

Consequently, the matrix $A$ satisfies to the algebraic quadratic Riccati equation

$$
A^{T} H_{1} A+A^{T} H_{2}+H_{2}^{T} A+H_{4}=0 .
$$

For the stated problem equation 13 has two nondegenerated solutions - the positively and negatively determined matrices $A$, corresponding respectively to $S_{l}^{+}$and $S_{l}^{-}$. They can be found arbitrarily precise. The generating function of $S_{l}^{+}$or $S_{l}^{-}$is $V_{l}(x)=\bar{x}\left(\frac{1}{2} A\right) \bar{x}^{T}+c$, where $c$ is a constant. It is convenient to take $c=0$ for the canonical transformations, preserving the origin $x=0$, and so, $\left.V_{l}(x)\right|_{x=0}=\left.V(x)\right|_{x=0}=0$.

\section{Proposition 1.}

For any parametrization $p: \mathbb{R}^{n} \rightarrow \mathbb{R}^{2 n}: s=\left(s_{1}, \ldots, s_{n}\right) \mapsto\left(x^{1}(s), \ldots x^{n}(s), V_{1}(s), \ldots, V_{n}(s)\right)$ of the separatrix $S_{l}^{+}$for the canonical linear case $\left(\Omega, I d \phi_{l}, S_{l}^{+}\right)$the following equations hold

$$
\left\{\begin{array}{l}
\left.(I d \phi) V\right|_{S^{+}}=\left.\left(I d \phi_{l}\right) V_{l}\right|_{S_{l}^{+}} \\
\left.(I d \phi)^{2} V\right|_{S^{+}}=\left.\left(I d \phi_{l}\right)^{2} V_{l}\right|_{S_{l}^{+}} \\
\cdots \\
\left.(I d \phi)^{2 n} V\right|_{S^{+}}=\left.\left(I d \phi_{l}\right)^{2 n} V_{l}\right|_{S_{l}^{+}}
\end{array}\right.
$$

They are implicite precise equations of $S^{+}$.

Proof. Both sides of equations 14 are created in an invariant way, applied to the corresponding triples $\left(\Omega, I d \phi_{l}, S_{l}^{+}\right) \sim\left(\Omega, I d \phi, S^{+}\right)$.

\section{An Example}

For the control system

$$
\left\{\begin{array}{l}
\dot{x}^{1}=\sin x^{2} \\
\dot{x}^{2}=\frac{\cos x^{1}}{\cos x^{2}} u
\end{array}\right.
$$

and functional

$$
J=\int_{0}^{\infty}\left(\left(x^{1}\right)^{2}+\left(\cos x^{1}\right)^{2} u^{2}\right) d t
$$

it is required to find the optimal stabilizing feedback $u_{\text {opt }}(x)$.

Applying the above technique we get the following.

$$
u_{o p t}=-\frac{1}{2 \cos x^{1} \cos x^{2}} V_{2}
$$

is the optimal feedback on $S^{+}$.

$$
\phi=\left(\sin x^{2}\right) V_{1}-\frac{1}{4\left(\cos x^{2}\right)^{2}}\left(V_{2}\right)^{2}+\left(x^{1}\right)^{2}
$$

is the Hamiltonian.

$$
I d \phi=\sin x^{2} \frac{\partial}{\partial x^{1}}-\frac{V_{2}}{2\left(\cos x^{2}\right)^{2}} \frac{\partial}{\partial x^{2}}-2 x^{1} \frac{\partial}{\partial V_{1}}+\left(\frac{\left(\sin x^{2}\right)\left(V_{2}\right)^{2}}{2\left(\cos x^{2}\right)^{3}}-\left(\cos x^{2}\right) V_{1}\right) \frac{\partial}{\partial V_{2}}
$$


is the Hamiltonian vector field.

$$
\begin{array}{r}
\phi_{l}=x^{2} V_{1}-\frac{1}{4}\left(V_{2}\right)^{2}+\left(x^{1}\right)^{2}, \\
I d \phi_{l}=x^{2} \frac{\partial}{\partial x^{1}}-\frac{1}{2} V_{2} \frac{\partial}{\partial x^{2}}-2 x^{1} \frac{\partial}{\partial V_{1}}-V_{1} \frac{\partial}{\partial V_{2}}
\end{array}
$$

are respectively the quadratic approximation Hamiltonian and linearized Hamiltonian vector field.

$$
V_{l}(x)=\sqrt{2}\left(x^{1}\right)^{2}+\sqrt{2}\left(x^{2}\right)^{2}+2 x^{1} x^{2}
$$

is the generating function of $S_{l}^{+}$in the linearized problem.

It can be checked for this particular case that

$$
V_{l}^{(n)}=\left(I d \phi_{l}\right)^{n}\left(V_{l}\right)=(-\sqrt{2})^{n} V_{l},
$$

where $V_{l}^{(n)}$ is the $n$-th derivative of function $V_{l}$ along the vector field $I d \phi_{l}$. So, to get invariant equations

$$
\left\{\begin{array} { l } 
{ I d \phi V = - \sqrt { 2 } V } \\
{ ( I d \phi ) ^ { 2 } V = 2 V } \\
{ ( I d \phi ) ^ { 3 } V = - 2 \sqrt { 2 } V }
\end{array} \quad \text { or } \quad \left\{\begin{array}{l}
(I d \phi)^{2} V=-\sqrt{2}(I d \phi) V \\
(I d \phi)^{3} V=2(I d \phi) V
\end{array}\right.\right.
$$

of $S^{+}$it is sufficient to compute just three derivatives.

Computing

$$
\left\{\begin{array}{l}
I d \phi(V)=\left(\sin x^{2}\right) V_{1}-\frac{\left(V_{2}\right)^{2}}{2\left(\cos x^{2}\right)^{2}} \\
(I d \phi)^{2} V=\frac{V_{1} V_{2}}{2 \cos x^{2}}-2 x^{1} \sin x^{2} \\
(I d \phi)^{3} V=-\frac{1}{2}\left(V_{1}\right)^{2}-2\left(\sin x^{2}\right)^{2}
\end{array}\right.
$$

one can obtain implicit equations of $S^{+}$for the given problem

$$
\left\{\begin{array}{l}
\frac{V_{1} V_{2}}{2 \cos x^{2}}-2 x^{1} \sin x^{2}=-\sqrt{2}\left(\sin x^{2}\right) V_{1}+\frac{\sqrt{2}\left(V_{2}\right)^{2}}{2\left(\cos x^{2}\right)^{2}} \\
-\frac{1}{2}\left(V_{1}\right)^{2}-2\left(\sin x^{2}\right)^{2}=2\left(\sin x^{2}\right) V_{1}-\frac{\left(V_{2}\right)^{2}}{\left(\cos x^{2}\right)^{2}} .
\end{array}\right.
$$

Since the solution of equations 25 of the form $V_{i}=\frac{\partial}{\partial x^{i}} V(x), i=1,2$, is unique it is sufficient to know just one arbitrary point $\left(x_{*}^{1}, x_{*}^{2}, V_{1 *}, V_{2 *}\right)$ satisfying the equations, express after the differentials $d V_{i}, i=1,2$, from the corresponding linear system, and approximately calculate the required surface.

For the given case an exact solution can be found with an appropriate substitution. The result is

$$
S^{+}:\left\{\begin{array}{l}
V_{1}=2 \sqrt{2} x^{1}+2 \sin x^{2} \\
V_{2}=2 x^{1} \cos x^{2}+2 \sqrt{2} \sin x^{2} \cos x^{2} .
\end{array}\right.
$$

The optimal stabilizing feedback is

$$
u_{\text {opt }}(x)=-\frac{x^{1}+\sqrt{2} \sin x^{2}}{\cos x^{1}}
$$

where $\left(x^{1}, x^{2}\right) \in W \subset\left\{\left(x^{1}, x^{2}\right) \mid-\frac{\pi}{2}<x^{1}, x^{2}<\frac{\pi}{2}\right\}$, the set $W$ is a maximal neighbourhood of $0 \in \mathbb{R}^{2}$, where $S^{+}$exists.

\section{Conclusion}

The method of invariants applied to the optimal stabilization problem reduces it to algebraic calculations and creates exact implicit equations of the separatrix surface where the optimal feedback function is determined.

Although, in a general case the obtained equations are hardly computable exactly, they admit computable linearization and computation by step of any point starting from one known. 


\section{References}

[1] G. V. Kondratiev, Geometric theory of synthesis of smooth optimal stationary control systems, Fizmatlit, Moscow, Russia, 2003.

[2] V. I. Arnold, A. Weinstein, K. Vogtmann, Mathematical methods of classical mechanics, Springer, 1989.

[3] F. W. Warner, Foundations of Differentiable Manifolds and Lie Groups, Springer, 1983.

[4] Élie Cartan, La méthode de repère mobile, la théorie des groupes continus, et les espaces généralisés, Hermann et Cie, Paris, 1935.

[5] Élie Cartan, La théorie des groupes finis et continus et la géométrie différentielle traitées par la méthode du repère mobile, Gauthiers-Villars, 1937.

[6] R. W. Sharpe, Differential Geometry, Cartan's Generalization of Klein's Erlangen Program, Springer, 1997.

[7] P. J. Olver, Equivalence, Invariants, and Symmetry, Cambridge University Press, 1995.

[8] G. V. Kondratiev, A Categorical-Informational Approach to the Value Prediction Problem, Atti della Accademia Peloritana dei Pericolanti: Classe di Scienze Fisiche, Matematiche e Naturali, Vol. 91, No. 2, A3, 11 p.p., URL: http://dx.doi.org/10.1478/AAPP.912A3, 2013.

[9] G. V. Kondratiev, Natural Transformations in Statistics, SciPress, International Frontier Science Letters, vol. 6, 2015. 